\title{
A method of intra-ventricular bioimpedance spectroscopy to estimate the dynamic volume of right ventricle
}

\begin{abstract}
Dynamic volume measurements of the heart are primarily done in the left ventricle, but as the right ventricle is a frequent place for pacing electrodes it could be easier to estimate cardiac output through the right ventricle. Our goal was to find the correlation between intra-cardiac bioimpedance deviations and the variations related to changes of the right ventricle volume. The variations of right ventricle volume have been evaluated experimentally using a setup with an isolated pig heart. Measurements were made with an intra-ventricular catheter and using multifrequency impedance spectroscopy.
\end{abstract}

Keywords: bioimpedance, cardiac output, right ventricle volume, estimation

\section{Introduction}

In Europe, $45 \%$ of all deaths are a result of cardiovascular disease, and the costs that derive from heart failure exceed the costs of all other diseases together [1]. Therefore, the motivation to develop a method to improve diagnostics and prevent heart-related problems is considerable. The importance of dynamic volume changes and the function of the right ventricle have increased in the last decade because they provide necessary information to assess cardiovascular performance [2]. Measuring blood volume changes in the right ventricle is essential to estimate total cardiac output and it is an important parameter to characterize the heart and its pump function in experimental studies. Cardiac output is usually measured from the left ventricle [3] because its geometry is relatively simple - a sphere - while the right ventricle is like an unequal layer around left ventricle in 3D space. Also, the right ventricle is thin-walled compared to the left ventricle, making the measurements difficult and more sensitive to changes from outside the right ventricle (Fig. 1).

\footnotetext{
Acknowledgement: This research was supported by the Estonian Research Agency grant IUT1911 and the European Regional Development Fund in frames of the Estonian Center of Research Excellence EXCITE. The authors thank SMARTIMPLANT Ltd.
}

Andres Kink, SMARTIMPLANT Ltd., Tallinn, Estonia, e-mail: andres@linking.ee Marek Rist, Raul Land, Hip Kõiv, Mart Min, Th. J. Seebeck Department of Electronics, Tallinn University of Technology, Tallinn, Estonia

https://doi.org/10.1515/9783110558920-017 


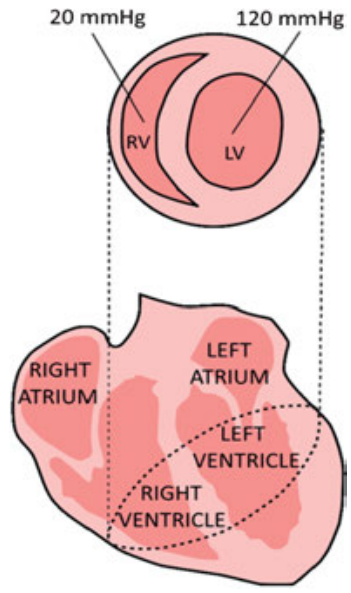

Fig. 1: Structure and shape of the adult right and left ventricles.

Although the left ventricle has a better shape for measurements, in real life the right ventricle is a basis for artificial pacing and a common place for pacing electrodes, thus making cardiac output estimation in the right ventricle potentially more convenient. Modern heart assisting devices like pacemakers are "smart devices" that can regulate the heart according to its condition. To help in the management of the unstable patient, measurements of cardiac output need to be taken repetitively [4]. Pacing electrodes are inside the right ventricle permanently, hence making constant measurements possible. Our main goal was to estimate the absolute or relative right ventricle volume changes experimentally with impedance spectroscopy and a multielectrode intra-ventricular catheter using a setup with an isolated pig heart. Multiple hypotheses have been developed and evaluated.

1) Bioimpedance can be used for the measurement of the amount of blood in the vicinity of the electrodes, however due to unknown position and shape (bending) of the electrode the measured result may not correlate with the actual volume change in the whole ventricle.

2) By using bioimpedance spectroscopy, the distance between the electrode and the cardiac tissue could be evaluated based on the changes in $\beta$ dispersion area, since impedance of blood has negligible reactive components in the used frequency range from $1 \mathrm{kHz}$ up to $349 \mathrm{kHz}$ and heart muscle has a much larger reactive component due to the dense packing of muscle cells [5].

3) With compensation based on the estimated position of the electrode, the segment-based volume changes could be measured with better precision.

The use of a multielectrode intra-ventricular catheter for impedance spectroscopy is a potentially useful technique for repetitive measurements to estimate the right ventricular volume. 


\section{Methods and general principles of design}

We evaluated the right ventricle volume during multiple working cycles with different loading conditions. Usually intra-cardiac impedance is measured with single frequency but we believe that by using multifrequency measurements, additional information could be gained. The change of volume is measured by inserting an Inquiry 1110-5-25-M decapolar electrophysiology catheter, shown in Fig. 3, from St. Jude Medical into the right ventricle and monitoring the change in impedance between the electrodes using the four-wire connection method. The electrode positioning is uneven: 2-5 mm, giving four 2-5-2 (distances between electrodes in $\mathrm{mm}$ ) measurement combinations or seven when we also include 5-2-5 combinations. Measured current and voltage values from 2-5-2 and 5-2-5 need separate correlation parameters when translating measured impedance data to blood volume (Fig. 2).

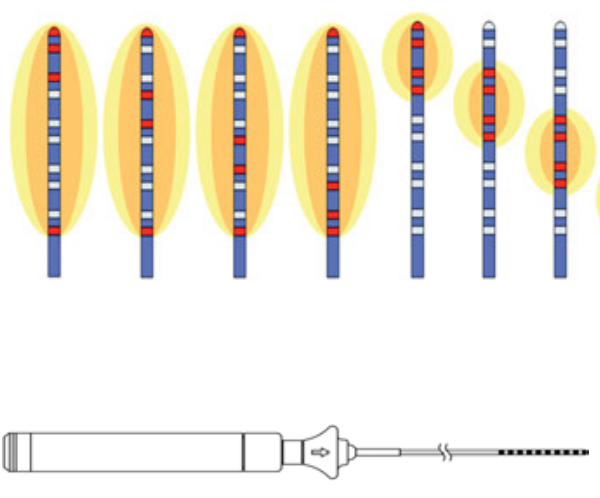

Fig. 3: Catheter that is inserted into the right ventricle through the pulmonary artery to measure impedance change (INQUIRYTM electrophysiology catheter).

The relationship between volume (V), electrode distance, impedance and blood resistivity is given as follows:

$$
V=\frac{1}{\alpha} \cdot \rho \cdot L^{2} \cdot\left(G_{x}-G_{p}\right)
$$

where $\rho$ is the resistivity of the blood, $L$ is the distance between measurement electrodes, $\alpha=\frac{\mathrm{SV}_{\text {conductance }}}{\mathrm{SV}_{\text {reference }}}$ is a stroke volume (SV) correction factor, $G_{\chi}$ is a total measured conductance and $G_{p}$ is the conductance of heart muscle tissue [3]. This equation is important for left ventricle volume measurements but research [6] has shown it is accurate also in the right ventricle. Our experiments show that it is not reliable to use the given equation inside the right ventricle due to the unknown electrode position. In order to obtain information about the shape and volume of the ventricle during operation of the heart, the measurement must be made relatively fast. When using a 
single frequency, fast measurement is relatively easy to achieve. When using spectrum measurement, obtaining real-time measurements becomes more difficult. We have developed a real-time impedance spectrum analysis device, Quadra, which uses binary multifrequency signals for excitation and measures 15 frequency points, from $1 \mathrm{kHz}$ to $349 \mathrm{kHz}$, with a period of $1 \mathrm{~ms}$, giving 1000 measurements per cardiac cycle when the heart rate is $60 \mathrm{bpm}$ [7].

Excitation in the form of voltage was used with $V_{\mathrm{RMS}}$ of $200 \mathrm{mV}$ resulting in root mean square (RMS) currents in the range of $0.8 \mathrm{~mA}$ to $1.2 \mathrm{~mA}$. Since we need to analyze four or seven sets of electrodes, a fast switching of the measurement device to electrode sets is needed. This could be done with 28 relays or one integrated crosspoint switch. The best results were achieved by using jumping excitation along with measurement. Changing excitation is related to longer stabilization time, thus reducing the measurement rate.

We noticed a large measurement noise at frequencies $<10 \mathrm{kHz}$ when multiplexing both excitation and sense electrodes. The noise decreases exponentially in time and can be considered negligible after $100 \mathrm{~ms}$ of settling time. This could be due to the formation of an electrical double layer since we are measuring in an electrolytic environment [8].

When the excitation was fixed to the first and last electrodes, and only the position of the sense electrodes was multiplexed, the measurement noise from lower frequencies was removed. The current distribution, in an already difficult environment, gets a lot more complex and interpretation of measured impedance would be more difficult.

For the study of our results we use custom-made analysis software made in LabVIEW. It shows impedance magnitude values, the pressure value and the phase angle in real time.

\section{Measurement setup}

For experiments, we obtained a pig's heart from the slaughterhouse, which was in the condition to be resuscitated. The electrophysiology catheter was placed inside the right ventricle through the pulmonary artery together with a silicone tube to manipulate the loading condition and the blood volume. In addition, a tube for the pressure sensor was added to measure the blood pressure in the right ventricle. The tricuspid valve was then closed to prevent the tubes from moving too much in the ventricle. The coronary arteries were kept connected for the external blood supply to keep the heart alive. Oxygenated warm blood was used for heart perfusion, since living heart needs oxygenated blood of a correct temperature (around $37^{\circ} \mathrm{C}$ ) (Fig. 4).

For measurements, an intra-ventricular catheter was attached to the real-time impedance spectrum device which was connected to the PC and analysis software made in LabVIEW (Fig. 5). The right ventricle was then filled with blood and the heart 


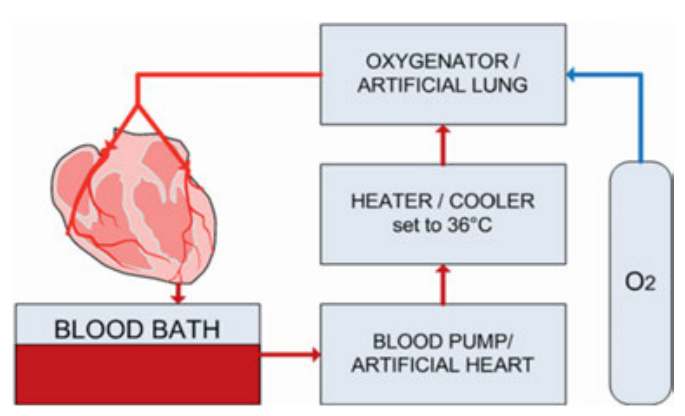

Fig. 4: Isolated retrogade perfusion heart setup.

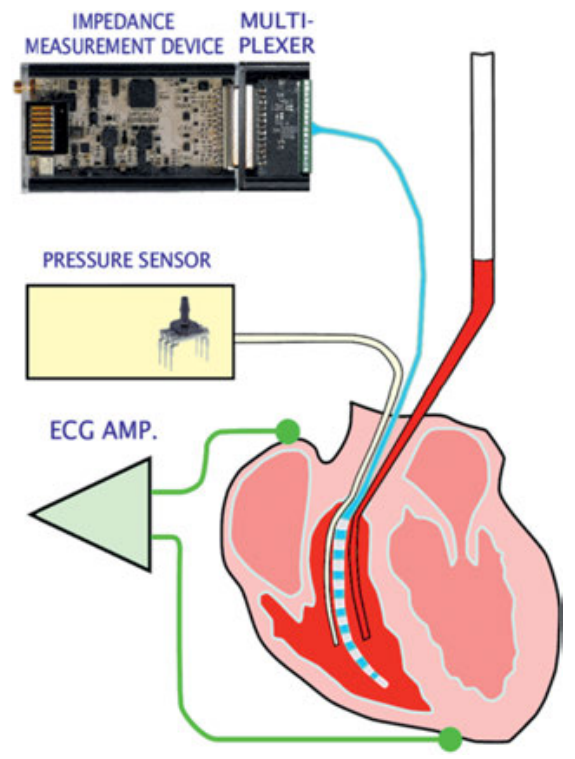

Fig. 5: Measurement setup with pressure sensor and impedance measurement device. ECG is useful to monitor heart electrical activity.

was given an electrical impulse or was contracted by manual squeezing to revive it. Changing the blood volume inside the right ventricle gave us impedance results we could expect. We used four-wire measurement. Method A consists of switching the excitation position and switching the sense position, which gives us a more uniform and localized current distribution but also more measurement noise. Method B consists of a fixed excitation position and switching the sense position, giving a shorter acquisition time but a wider and less uniform current distribution.

\section{Results}

A versatile, compact and portable laboratory was constructed for isolated experiments together with measurement devices. The isolated heart filling was modulated accord- 
ing to the level in the tube inserted into the pulmonary artery. The pulmonary valve was removed to adapt different loading conditions in the right ventricle. Under low loading conditions, prominent changes in bioimpedance were registered and evaluated in tissue distance changes. Our research shows that phase angle differences were the most noticeable. Under mean or high loading conditions, variations in the phase angle do not correlate well with value changes inside the right ventricle.

The initial position and changes in position of the electrodes can be detected with bioimpedance measurements. It can be seen from Fig. 6 that the modulus of measured

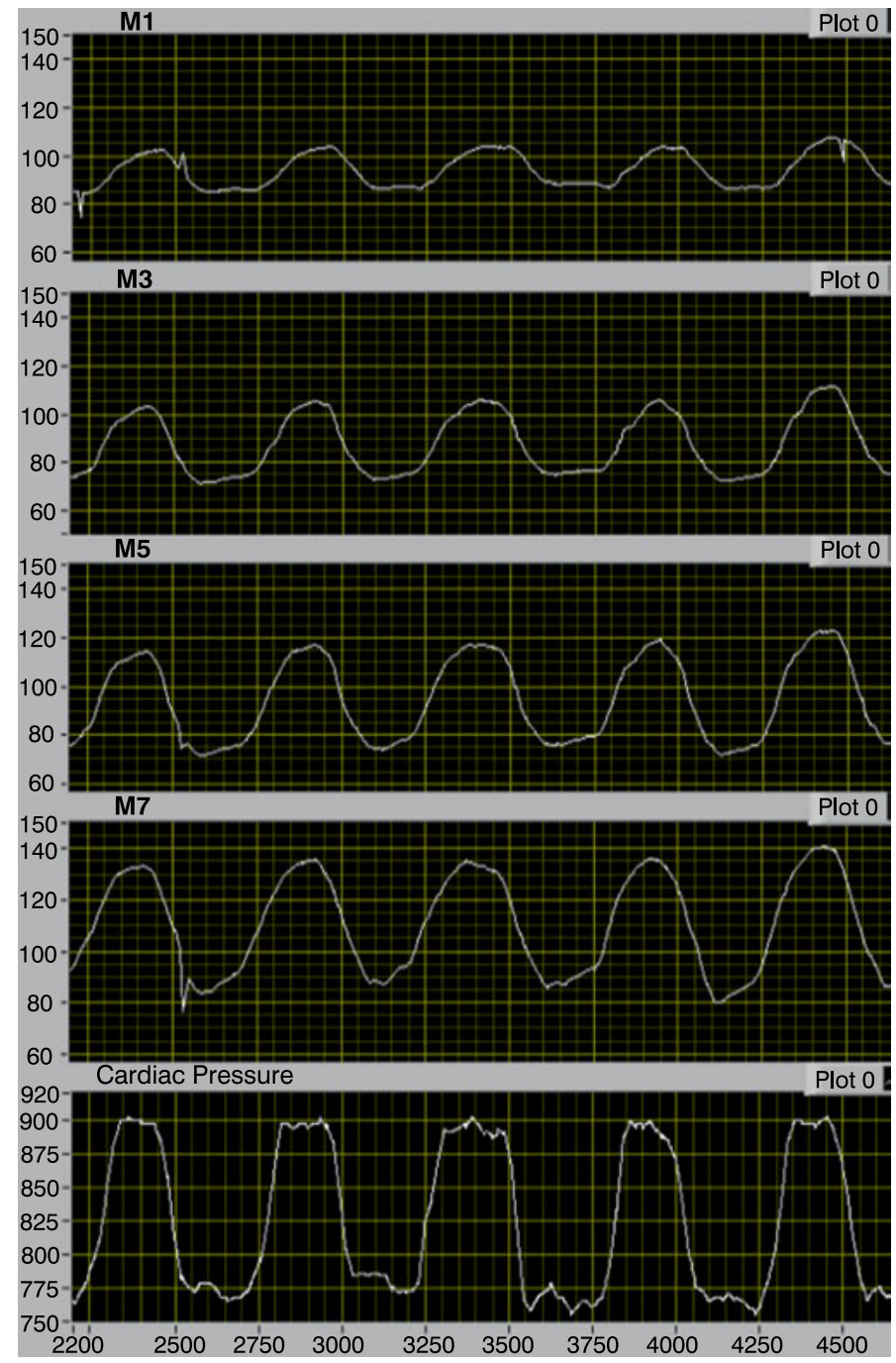

Fig. 6: Magnitude (M1, M3, M5, M7 in Ohms) and pressure (in $\mathrm{mmHg}$ ) curves measured from the right ventricle. 

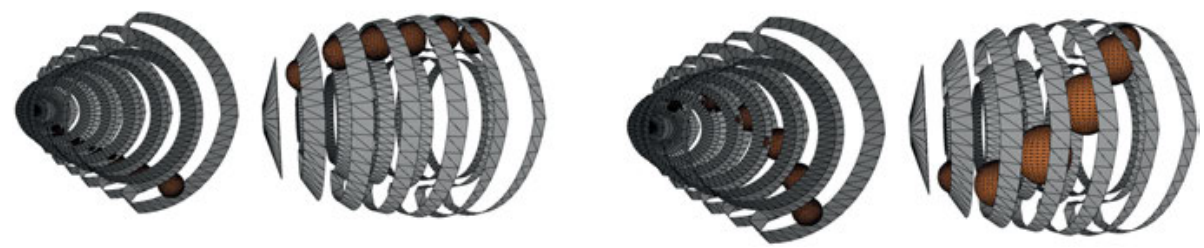

Fig. 7: Electrode positions 1 and 2 with sensitivity spaces (spheres).
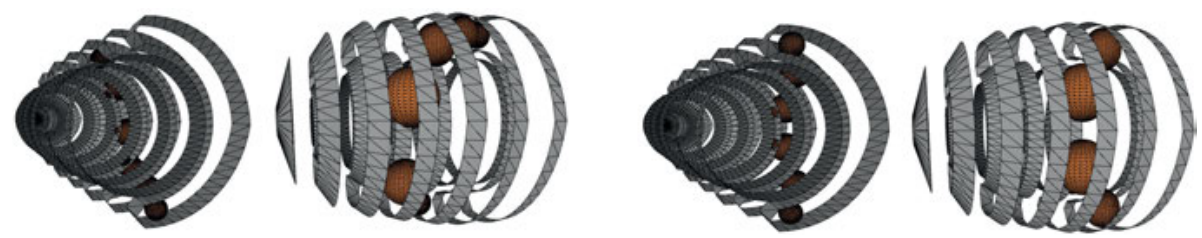

Fig. 8: Electrode positions 3 and 4 with sensitivity spaces (spheres).

impedance shows good correlation with the cardiac pressure. The changes in magnitude of different segments represent the volume of blood and the proximity of the myocardium. We are able to assess segment-based volume changes in the right ventricle. Fig. 7 and Fig. 8 show segments of the heart and how electrode positions can change during the heart cycle. Four different positions are proposed with spheres marked as electrode sensitivity spaces.

\section{Discussion}

In the present study, we have examined blood volume changes in the right ventricle using impedance spectroscopy and a decapolar catheter. We measured electrode shape changes, segment-based volume changes and the distance between the measurement electrode and the cardiac muscle tissue in the right ventricle by using bioimpedance measurements with an intra-ventricular electrophysiological catheter. The proposed technique is affected by the complex geometry of the right ventricle and the conductivity of surrounding tissues. Using a Baan catheter [3] there is an assumption that the electric field produced by the catheter is homogeneous, parallel to the long axis of the ventricle, and does not extend to other cardiac chambers. If these presumptions are not considered, the errors of ventricle volume estimation are significant. The outcome shows a noticeable correlation between the changes of bioimpedance and the variations of right ventricle volume, but the interpretation of results can be quite complicated due to the different electrode positions inside the right ventricle. For acceptable results, it is necessary to estimate the electrode configuration inside the right ventricle 
and separate impedance change contributions from blood and cardiac tissue. This is a subject of ongoing research.

\section{Bibliography}

[1] ESC, “European cardiovascular disease statistics 2017 edition”, 2017.

[2] W. H. W. Tang and W. Tong, "Measuring impedance in congestive heart failure: Current options and clinical applications", American Heart Journal, 2009.

[3] J. Baan, T. Aouw Jong, P. L. M. Kerkhof, R. J. Moene, A. D. Van Dijk, E. T. Van Der Velde, and J. Koops, "Continuous stroke volume and cardiac output from intra-ventricular dimensions obtained with impedance catheter", Cardiovascular Research, 1981.

[4] M. R. Pinsky, "Why measure cardiac output?", Critical Care, 2003.

[5] P. A. White and A. N. Redington, "Right ventricular volume measurement: Can Conductance do it better?”, Physiological Measurement, 2000.

[6] H. Helmholtz, "Ueber einige Gesetze der Vertheilung elektrischer Ströme in körperlichen Leitern mit Anwendung auf die thierisch-elektrischen Versuche", Annalen der Physik und Chemie, 1853.

[7] M. Rist, M. Reidla, R. Land, T. Parve, O. Märtens, P. Annus, J. Ojarand, and M. Min, "Modular system for spectral analysis of time-variant impedances", In: IFMBE Proceedings, 2013.

[8] S. Grimnes and $\emptyset$. G. Martinsen, "Bioimpedance and Bioelectricity Basics", Academic Press, 2000. 


\title{
Meat quality monitoring by classification based on impedance spectra
}

\begin{abstract}
In this contribution, we investigate the possibilities of meat state assessment based on bioimpedance spectroscopy and fuzzy methods. We report on the procedure of feature selection from impedance spectra of meat aiming to reduce measurement data to features providing sufficiently information to build the basis for meat state estimation, classification of meat type and estimation of the level of freshness. The developed method is suitable for signal processing of impedance spectra in a devices for real-time meat monitoring based on bioimpedance spectra.
\end{abstract}

Keywords: impedance spectroscopy, bioimpedance, fuzzy logic, feature extraction, data mining

\section{Introduction}

Meat quality cannot be measured directly, like a classical measurement quantity. It includes different aspects related to microbial attacks, the existence of certain substances and hormones and structural changes of the muscle during maturation processes. Especially for beef, maturation and structural muscle changes are important to realize a tender state by consumption. In order to follow muscle strutural changes, electrical measurement methods, such as bioimpedance. Bioimpedance spectroscopy is fast, non destructive and can be realized at low-cost.

Several investigations have been carried out in the field of meat and food diagnosis. Different features of the impedance spectra can be adopted for data mining such as the use of model parameters, the use of characteristic points, the extraction of data at a fixed frequency or the use of the whole measurement results. An overview is given about different results realized with data mining and classification methods related to the topic of biological tissue monitoring.

Guerrero et al. [1] have used Cole-Cole model parameters to describe the relationship between sensory properties and electrical parameters of different meat qualities of hams for the semimembranosus (SM) and biceps femoris (BF) muscles. The used parameters are $R_{0}$, which corresponds to the electrical impedance at lower frequencies,

Mahdi Guermazi, Chair for Measurement and Sensor Technology, Chemnitz University of Technology, Reichenhainerstrasse 70, 09126 Chemnitz, Germany; and National School of Engineers of Sfax, University of Sfax, Sfax, Tunisia, e-mail: mahdienis@yahoo.fr

Olfa Kanoun, Chair for Measurement and Sensor Technology, Chemnitz University of Technology, Reichenhainerstrasse 70, 09126 Chemnitz, Germany

Nabil Derbel, National School of Engineers of Sfax, University of Sfax, Sfax, Tunisia

https://doi.org/10.1515/9783110558920-018 
$R_{\text {inf }}$, which corresponds to the electrical impedance at high frequencies, $\alpha$, which corresponds to the shape adjustment parameter, and $f_{c}$, which corresponds to the characteristic frequency of the region under measurement at which the imaginary part of the electrical impedance has the largest absolute value. A fifth parameter is introduced: the ratio between $R_{\text {inf }}$ and $R_{0}$, which is proportional to the ratio of extracellular water to total water in meat. Principal component analysis is used in order to group different hams and to locate them in the biplot. They are classified in three groups according to the main components: hams having $\mathrm{pH} 45$ (defined as the $\mathrm{pH}$ at $45 \mathrm{~min}$ postmortem) lower than 5.85 were classified as pale, soft and exudative (PSE), hams having $\mathrm{pHu}$ (defined as the $\mathrm{pH}$ at $24 \mathrm{~h}$ postmortem) higher than 6.0 as dark, firm and dry (DFD) and the rest as normal hams. The electrochemical impedance spectroscopy (EIS) prototype correctly detected $69.2 \%$ and $56.0 \%$ for SM and BF muscles, respectively, of the problem hams in terms of pastiness. We suggest that the electrical parameters evaluated in green hams by the EIS prototype could be useful for predicting pastiness in dry-cured ham.

Also, for the application of bovine tissue classification, Negri et al. [2] have used features for classification composed by the Cole-Cole parameters $R_{0}, \operatorname{Ro}, \tau$ and $\alpha$. Results show that using the fitted Cole-Cole model parameters instead of the full spectrum as the input of a neural network can enhance its classification rate and significantly reduce its topology when tested with experimental bovine tissue spectra.

The same method has been used by Filho et al. [3] for classification to assess bovine milk quality. They have confirmed the use of the Cole-Cole parameters may have a better classification than the use of the raw acquired spectrum. Using a multilayer perceptron (MLP), the recognition percentage can reach $94.6 \%$ to classify adulterated milk samples with the type of impurity added being water $\left(\mathrm{H}_{2} \mathrm{O}\right)$ or hydrogen peroxide $\left(\mathrm{H}_{2} \mathrm{O}_{2}\right)$. The used MLP is composed by a hidden layer with two neurons, an output layer with three neurons defining each class (milk, milk with $\mathrm{H}_{2} \mathrm{O}$, milk with $\mathrm{H}_{2} \mathrm{O}_{2}$ ).

Meat monitoring is done using the three main parts: the experimental investigations, the modeling and features analysis and finally the meat type and freshness diagnosis (Fig. 1).

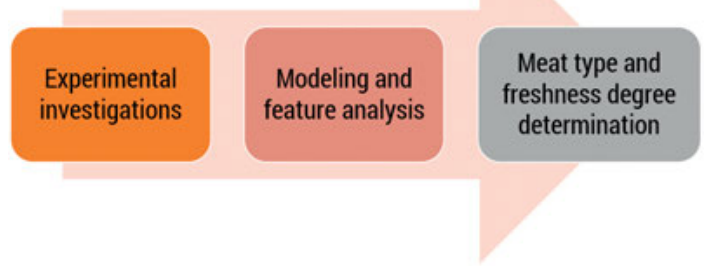

Fig. 1: Monitoring process of meat quality. 


\section{Methodology}

The most important and necessary information from a meat monitoring device is the type of the muscle and the freshness of the meat. To ralize a suitable signal processing, it is necessary to remove spikes in the measurements due to errors. Another important factor that should be respected is the use of a reduced input in order to reduce the effort and increase the stability of implementation. The process of classification is therefore composed of two principal steps:

- Accurate feature selection from the measured impedance spectra;

- Classification with high reliability.

\subsection{Feature selection}

Before classfication, generally feature selection should be carried out to reduce the measured data to specific data, which serve as the basis for classification. Selection of features represents therefore a decisive step in order to reach a high classification probability during learning and test phases. Measurements have been carried out with different tissues and for a long period of time in order to monitor the degradation state with time. The measurement setup consists of a laboratory impedance analyzer (Agilent 4294 A) connected to a personal computer for data acquisition. The spectrometer generated a signal within a frequency band ranging from $40 \mathrm{~Hz}$ to $110 \mathrm{MHz}$ (Fig. 2). Cylindrical penetrating multielectrodes are used for measurements ensuring the reduction of the anisotropy effect and good contacts between electrodes and the tissue [4].

There are different possibilities for selecting features from impedance spectra, such as the use of model parameters, the use of characteristic points, the extraction

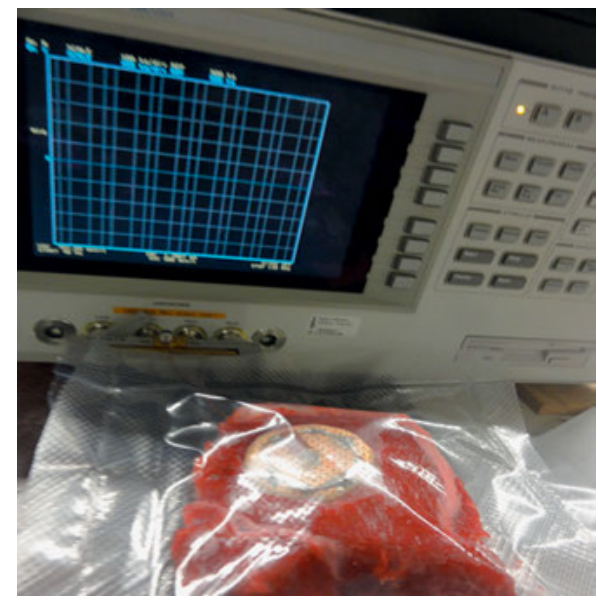

Fig. 2: Meat measurement. 
of data at a fixed frequency or the use of the whole measurement results. According to literature and to experimental investigations, measurement results have been evaluated by means of the modified Fricke-Cole-Cole model. Results are reproducible and correspond to the expected behavior due to aging [5]. The model parameter evolution shows principally the same behavior, but differs from one muscle to another in the absolute value. This is due to several factors, especially the biologic characteristics of each muscle.

Both model parameters (MP) and characteristic points (CP) can be principally used for data mining and represent an accurate feature gathered from the measured impedance. The investigated possibilities are MP, CP and MP + CP (Fig. 3).

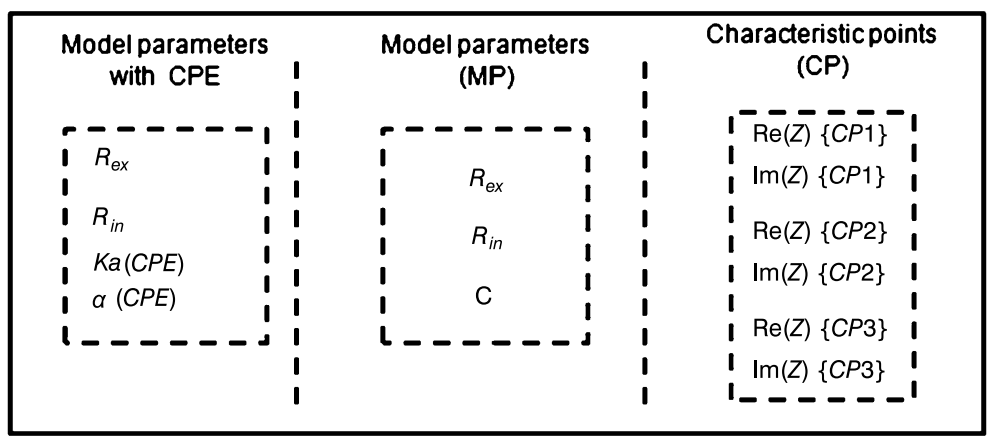

Fig. 3: Selected examples for feature extraction from impedance spectra.

According to the literature, principal component analysis (PCA) has been often used to reduce features to only the necessary inputs [6-8]. PCA belongs to statistical methods of multivariate analysis [9]. It is a method of transforming the initial dataset of high dimensions into a new dataset represented by a vector of samples. The goal of this transformation is to concentrate the information and reduce its dimension by considering the data variance. The PCA method is a linear transformation, as described in equation (1), which transfers the original possibly corresponding data set $X$ to a new uncorrelated dataset $F_{\text {new. }}$. The new dataset $F_{\text {new }}$ has the same dimension as the original dataset $X ; A_{t}$ is the coefficient transformation matrix. We have

$$
F_{\text {new }}=A_{t} X
$$

The first few components (columns) of the new dataset $F_{\text {new }}$ contribute the majority of variances and are considered to be the principal components, which can reflect the original data matrix without big information losses. They have smaller dimension and are uncorrelated to each other, which makes the analysis of the data much easier. The input data set $X_{d}$ is in the form of a matrix, as shown in equation (2), where $p$ is the 
number of the parameters and $n$ is the number of the observations. We have

$$
X_{d}=\left(\begin{array}{lll}
X_{11} & X_{12} & X_{1 p} \\
X_{21} & X_{22} & X_{2 p} \\
X_{n 1} & X_{n 2} & X_{n p}
\end{array}\right)
$$

The first two components for the feature composed by MP, for the $\mathrm{CP}$ and for the $\mathrm{MP}+\mathrm{CP}$ explain more than $90 \%$ of the variances. In order to verify which feature we should use for classification, a simple idea is used consisting of the principle of superposition of the test data in the corresponding region of input data after k-nearest neighbors (KNN) calculation. The algorithmic method of KNN was used to give a complete 2D presentation in a geometrical plot of the data.

\subsection{Classification method}

Different suitable methods of classification can be used in this application. Fuzzy logic is selected as a classification method that provides a successful alternative for easy classification, as it is in general a robust method. It has also the advantages to deal with uncertainty problems and usually gives good results for various areas. Fuzzy logic is widely used in medical applications for diagnosis of tuberculosis, cancer, asthma, diabetes, aphasia, malaria, HIV, pulmonary embolism, cortical malformations and pancreatic diseases [10]. Fuzzy logic has been implemented for an earlier detection of breast cancer [11, 12]. Results are found to be very useful and helpful for oncologists, radiologists and doctors. Thus, fuzzy logic, as an intelligent method, will be of great help especially when saving patient lives is possible. The process of fuzzy logic based classification includes three principal steps [13]:

- Fuzzification of input variables and choice of suitable membership functions;

- Construction of fuzzy rules and making decisions;

- Defuzzification.

An explanatory illustration of the fuzzy inference process is shown in Fig. 4.

In fact, the model parameters (received data) undergo a feature's extraction process (information), which is a preparatory step for the classification method. The resulting data are then fuzzified using membership functions. Afterward, fuzzy values are analyzed using fuzzy rules to get the fuzzy outputs. After defuzzification, the new data are then compared and classified with similarities to get the desired clusters.

Fuzzification is the conversion of crisp input data into fuzzy values or linguistic variables using membership functions. These fuzzy values reflect the human perspective of the given system. These fuzzifiers are membership functions determined depending on a subjective view of the problem and an individual's perception of the situation. Depending on the desired class and performances, simple functions are used to build membership functions, such as triangular, trapezoidal and Gaussian ones. 


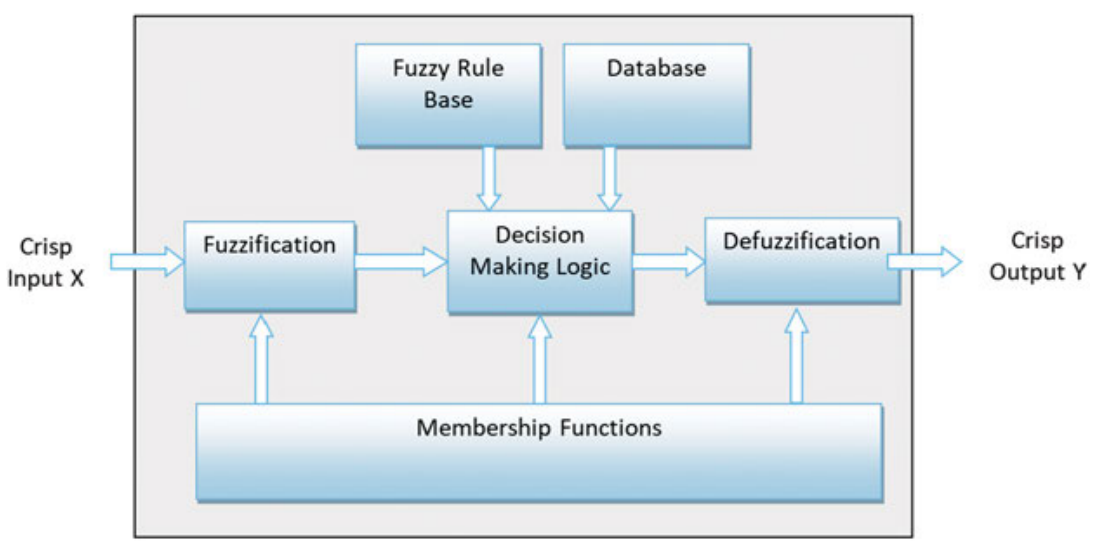

Fig. 4: Block structure of a Fuzzy system.

Fuzzy rules, which have the general form of "IF X is A THEN Y is B", are destined to map the fuzzy input $X$ to the fuzzy output $Y$. In a logical context, $X$ is referred to be a linguistic variable and A is a linguistic value. The 'IF' part of the rule is called antecedent; the 'THEN' part is called consequent. Connection between the rules is achieved using logical operators AND, OR and NOT. AND or min takes always the minimum of the functions value. OR (max) takes the maximum of the presented functions.

Defuzzification is the conversion of a fuzzy set quantity to a precise and deterministic set quantity. This step is necessary because in several applications, where the implementation is required, machines could only work with crisp or binary values and not with linguistic variables.

After defuzzification, the new data are compared and classified to get desired clusters [14]. Two important types of fuzzy logic systems exist and are much used for diagnosis: Mamdani inference [15, 16] and Takagi-Sugeno (TS) inference [17].

The Mamdani method is suitable for nonlinear systems, characterized by a fuzzy set consequent in the fuzzy rule that has the following form:

$\mathrm{R}: \mathrm{IF} \mathrm{L}\left(x_{1}\right.$ is $A_{1}, \ldots ., s_{k}$ is $\left.A_{k}\right)$ THEN $\left(y_{1}\right.$ is $B_{1}, \ldots ., y_{n}$ is $\left.B_{k}\right)$, where:

- $\quad x_{1}, . ., x_{k}$ are the inputs of membership functions;

- $A_{1}, . ., A_{k}$ are the fuzzy sets of the antecedent part;

- $\quad y_{1}, \ldots, y_{n}$ are the outputs of the membership functions;

- $B_{1}, \ldots, B_{n}$ are the fuzzy sets of the consequent part and $\mathrm{L}$ is the logical function connecting the propositions.

For the TS method, the output membership function or more precisely the rule consequent is only linear or constant. If the rule consequent is constant we call the model a zero-order Sugeno model, which has the following form:

$R_{0}: \operatorname{IF~L}\left(x_{1}\right.$ is $A_{1}, \ldots, s_{k}$ is $\left.A_{k}\right)$ THEN $y=k$,

where $k$ is a crispy constant defined by the operator. 
If the rule consequent is a linear function the Sugeno model is called a first-order Sugeno model with the following expression:

$R_{1}$ : IF L $\left(x_{1}\right.$ is $A_{1}, \ldots, s_{k}$ is $\left.A_{k}\right)$ THEN $y=f\left(x_{1}, \ldots x_{n}\right)$.

\section{Results and discussion}

\subsection{PCA computing}

A database composed of six muscles is used in order to use the PCA method well. This database will serve to determine the suitable feature. Three different muscles of two animals of different ages are used. The test data should be unknown. For that, two spectra will be used for learning and two others for testing in every case.

Singular-value decomposition is chosen following the study done in [18]. The decomposition of the normalized matrix " $\mathrm{X}$ " is done according to

$$
X^{\prime \prime}=\mathrm{USV}^{\prime}
$$

where $S$ is the $n \times p$ diagonal matrix with singular values $\sigma_{i}$ of the matrix $\mathrm{X}$ in diagonal form. We also have

$$
S_{i i}=\sigma_{i}
$$

and singular values are already sorted in a decreasing order, i. e.,

$$
\sigma_{1}>\sigma_{2}>\sigma_{2}>>\sigma_{p}
$$

The relationship between the singular value $\sigma_{i}$ and the eigenvalue $\lambda$ is given by

$$
\sigma_{i}=\sqrt{\lambda_{i}}
$$

where $U$ is the $n \times n$ left singular-valued vector matrix of $\mathrm{X}, V$ is the $p \times p$ right singularvalued matrix of $X$ and both are orthogonal matrices. The principal components $F$ can be calculated by equation (7), i. e.,

$$
F=X . V=U . S \cdot V^{\prime} . V=U . S .
$$

Before dealing with PCA computing, the inputs and test data of the three cases, MP, CP and MP + CP are defined. The test data have exactly the same dimension as the input data.

The result of the superposition of model parameter test data on the KNN partition corresponds to an accuracy equal to $100 \%$, as shown in Fig. 5 . The accuracy for the characteristic points is $66 \%$ with four erroneous outputs. For the feature composed 


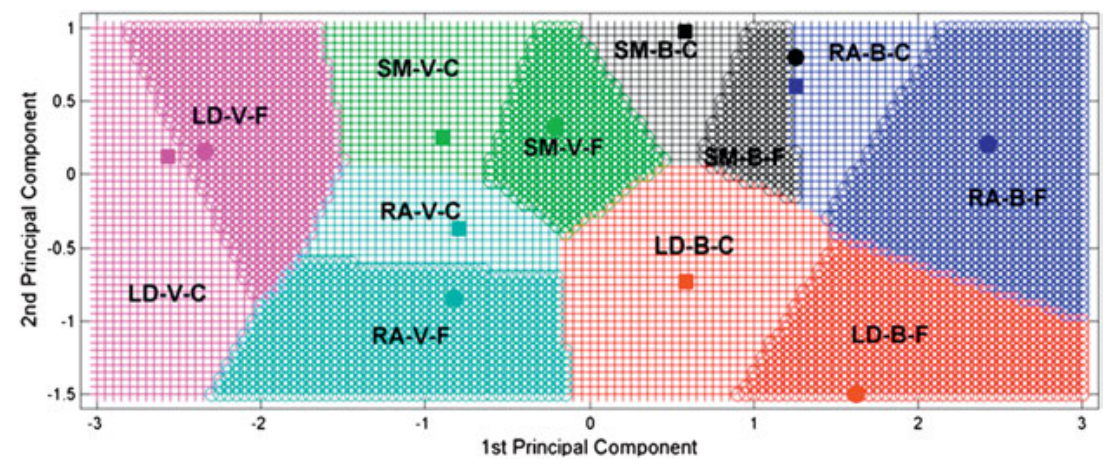

Fig. 5: Superposition of model parameter test inputs to the KNN graph.

by the characteristics points and model parameters we get 12 correct outputs that correspond to an accuracy of $100 \%$.

Feature extraction based on the use of model parameters and characteristic points shows good results but with the consideration of the use of reduced inputs in the network we choose to work with the model parameters only for the classification that also gives an accuracy of $100 \%$.

\subsection{Zero-order Sugeno model}

The classification is carried out using the TS method, which is similar to the Mamdani model in fuzzifying the inputs and applying the fuzzy operator. The major reason of this choice is that the TS method output membership can be either constant (zeroorder) or linear (first-order), corresponding exactly to the application specifications. The zero-order TS method with constant output memberships is exactly the solution that permits to get an accurate diagnosis and that corresponds to the problematic. For each muscle we appoint a corresponding constant number:

- 1 for the muscle LD beef (M1);

- 2 for the muscle RA beef (M2);

- 3 for the muscle SM veal (M3);

- 4 for the muscle LD veal (M4).

And for each muscle we appoint a corresponding constant number as output:

- 1 for fresh meat $(\mathrm{F})$;

- 2 for edible meat $(\mathrm{E})$;

- 3 for critical meat (C);

- 4 for dangerous meat (D). 


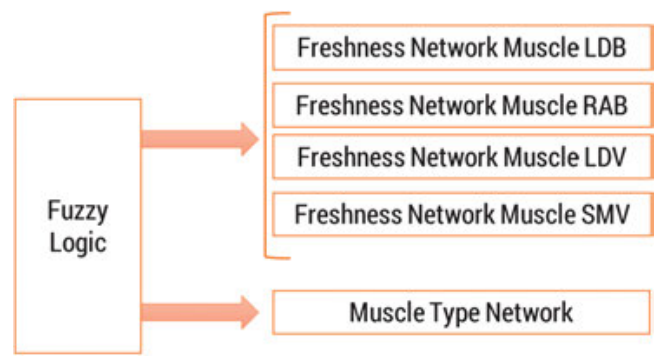

Fig. 6: Fuzzy logic process.

The fuzzy logic process (Fig. 6) is composed of two fuzzy logic systems that have been used for meat diagnosis; the first for the classification of the muscle type, the second for finding the freshness, to classify the meat in four classes that correspond to four different periods of time: fresh meat, edible meat, critical meat and dangerous meat.

The output membership function or more precisely the rule consequent is only constant. We have

$R_{0}: \operatorname{IF~L}\left(x_{1}\right.$ is $A_{1}, \ldots, s_{k}$ is $\left.A_{k}\right)$ THEN $y=k$.

\subsubsection{Membership functions}

The first step of the muscle type classification consists of the choice of the input and output variables and selecting carefully their intervals' ranges. By this step, we try to collect the data which generate the same output in one interval range. This process is carried out for all the inputs. In one side, we define the three model parameters $R_{e}$, $R_{i}$ and $C$, extracted from bioimpedance measurements as the inputs [5]; the output corresponds to the muscles $\mathrm{LD}_{\text {Beef }}, \mathrm{RA}_{\text {Beef }}, \mathrm{SM}_{\text {Veal }}$ and $\mathrm{LD}_{\text {Veal }}$. After several trials for the muscle type classification, we select the Gaussian membership functions (MF) defined by a central value $c$ and a standard deviation $\sigma>0$. We have

$$
\mu_{A}(x)=f(x, \sigma, c)=e^{\frac{-(x-c)^{2}}{2 \sigma^{2}}} .
$$

For each of the three inputs $R_{e}, R_{i}$ and $C$, we attribute the Gaussian membership functions associated with the intervals' ranges extracted from the database. It is necessary to use different zones for each input due to the performance of the learning. We attribute five membership degrees to the inputs $R e$ and $C$ and three to the input $R_{i}$. For each input the corresponding number of MF that leads to a high accuracy and that avoids the overlapping for the hole database represents the type of the meat.

For the fuzzy logic of freshness classification, the major difference with the fuzzy logic of muscle classification is that we do the learning for each muscle separately in order to determine the aging that corresponds to its freshness. The three model parameters $R_{e}, R_{i}$ and $C$ are defined as inputs and affected the trapezoidal MF after several trials. 
The fuzzy logic classification results show an accuracy of $100 \%$. The result of each muscle is in the close interval of the corresponding value; for example, for the veal $\mathrm{SM}$, the accepted result that corresponds to this muscle should be in the range from 2.5 to 3.5.

The fuzzy logic classification for noisy inputs reaches the same results as the used inputs. In the diagnosis process, the choice of confidence areas is decisive in order to support the learning process. We select and define the period of time such as:

- fresh, between day 2 and day 3;

- edible, between day 4 and day 6;

- critical, between day 7 and day 10;

- dangerous, more than day 11.

According to the defined periods, the accuracy is $84.62 \%$ for the beef LD, $92.31 \%$ for the beef RA, $100 \%$ for the veal SM and $61.54 \%$ for the veal LD.

\section{Conclusion}

Feature extraction using model parameters leads to a high accuracy. Suitable data for meat diagnosis are able to correctly classify muscle types and states. Fuzzy logic allows for an easy method of classification. It correctly classifies the inputs to the corresponding classes for the first step of meat type with a recognition index equal to $100 \%$.

For the freshness classification, fuzzy logic reaches a recognition index equal to $84.62 \%$ for the beef LD, $92.31 \%$ for the beef RA, $100 \%$ for the veal SM and $61.54 \%$ for the veal LD.

Fuzzy logic has many advantages which can be beneficial in the monitoring process of meat quality. These advantages are especially the consideration of noisy inputs, the tolerance that leads to a compact program according to the conception procedure and finally the simplicity.

\section{Bibliography}

[1] L. Guerrero, I. Gobantes, M.A Oliver, J. Arnau, M. D. Guardia, J. Elvira, P. Riu, N. Grebol, and J. M. Monfort, "Green hams electrical impedance spectroscopy (EIS) measures and pastiness prediction of dry cured hams", Meat Science, vol. 66, no. 2, pp. 289-294, 2004.

[2] L. H. Negri, P. Bertemes-Filho, and A. S. Paterno, "Computational intelligence algorithms for bio-impedance-based classification of biological material”, In: 5 th European Conference of the IFMBE, IFMBE Proceedings, vol. 37, pp. 1229-1232, 2011. 
[3] P. Bertemes-Filho, L. H. Negri, and A. S. Paterno, "Detection of bovine milk adulterants using bioimpedance measurements and artificial neural network", In: 5th European Conference of the IFMBE, IFMBE Proceedings, vol. 37, pp. 1275-1278, 2011.

[4] M. Guermazi, O. Kanoun, and N. Derbel, "Reduction of anisotropy influence and contacting effects in in-vitro bioimpedance measurements", Journal of Physics Conference Series, vol. 434, pp. 1-4, 2013.

[5] M. Guermazi, O. Kanoun, and N. Derbel, "Investigation of long time beef and veal meat behaviour by bio-impedance spectroscopy for meat monitoring”, IEEE Sensors Journal, vol.14, no. 10, pp. 3624-3630, 2014.

[6] V. Yu. Musatov, V. V. Sysoev, M. Sommer, and I. Kiselev, "Assessment of meat freshness with metal oxide sensor microarray electronic nose: A practical approach", Sensors and Actuators B, Chemical, vol.144, no.1, pp. 99-103, 2010.

[7] T. Eklöv, G. Johansson, F. Winquist, and I. Lundström, “Monitoring sausage fermentation using an electronic nose”, Journal of the Science of Food and Agriculture, vol. 76, no. 4, pp. 525-532, 1998.

[8] J. A. Balejko, N. Zbigniew, and B. Edyta, "Artificial neural network as the tool in prediction rheological features of raw minced meat", ACTA Scientiarum Polonorum. Technologia Alimentaria, vol. 11, no. 3, pp. 273-281, 2012.

[9] I. T. Jolliffe, "Principal Component Analysis", 2nd edition, Springers Series in Statistics, Springer, 2002.

[10] V. Prasath, N. Lakshmi, M. Nathiya, N. Bharathan, and N. P. Neetha, "A Survey on the applications of fuzzy logic in medical diagnosis", International Journal of Scientific \& Engineering Research, vol. 4, no. 4, pp.1199-1203, 2013.

[11] A. A. E. Saleh, S. E. Barakat, and A. A. E. Awad, “A fuzzy decision support system for management of breast cancer”, International Journal of Advanced Computer Science and Applications, vol. 2, no. 3, pp. 34-40, 2011.

[12] K. C. Latha, B. Madhu, S. Ayesha, R. Ramya, R. Sridhar, and S. Balasubramanian, "Visualization of risk in breast cancer using fuzzy logic in Matlab environment", International Journal of Computational Intelligence Techniques, vol. 4, no. 1, pp.114-117, 2013.

[13] H. J. Zimmermann, “Fuzzy Set Theory and Its Applications”, ISBN 978-94-015-7951-3, London, 1991.

[14] A. M. Aziz, "Effects of fuzzy membership function shapes on clustering performance in multisensor-multitarget data fusion systems", In: IEEE International Conference on Fuzzy Systems, pp.1839-1844, Korea, 2009.

[15] E. H. Mamdani and S. Assilian, "An experiment in linguistic synthesis with a fuzzy logic controller”. International Journal of Man-Machine Studies, vol. 7, no. 1, pp. 1-13, 1975.

[16] E. H. Mamdani, "Advances in the linguistic synthesis of fuzzy controllers", International Journal of Man-Machine Studies, vol. 8, no. 6, pp.669-678, 1976.

[17] T. Takagi and M. Sugeno, "Fuzzy identification of systems and its applications to modeling and control”, IEEE Transactions on Systems, Man and Cybernetics, vol. SMC-15, no. 1, pp. 116-132, 1985.

[18] F. Zhang, "Feature Extraction for the Meat's Freshness Characterization Using the Method of Principal Component Analysis”, Master of Science, TU Chemnitz, 2014. 
